Supporting Information for

\title{
Molecular Concentration Determination Using Long-Interval Chemical Exchange Inversion Transfer (CEIT) NMR Spectroscopy
}

\author{
Weiping Jiang ${ }^{1,2, \|,}$ Qianni Guo ${ }^{1,2, \|}$, Qing Luo ${ }^{1}$, Xiaoxiao Zhang ${ }^{1}$, Yaping Yuan ${ }^{1,2}$, Haidong Li $^{1,2}$ and \\ Xin Zhou ${ }^{1,2^{\star}}$ \\ ${ }^{1}$ Key Laboratory of Magnetic Resonance in Biological Systems, State Key Laboratory of Magnetic \\ Resonance and Atomic and Molecular Physics, National Center for Magnetic Resonance in Wuhan, \\ Wuhan Institute of Physics and Mathematics, Innovation Academy for Precision Measurement \\ Science and Technology, Chinese Academy of Sciences -Wuhan National Laboratory for \\ Optoelectronics, Wuhan 430071, People's Republic of China. \\ 2 University of Chinese Academy of Sciences, Beijing 100049, People's Republic of China. \\ " Weiping Jiang and Qianni Guo contributed equally to this work. \\ *Corresponding author: xinzhou@wipm.ac.cn
}

\section{List of Supplementary Content \\ Experimental description \\ Supplementary Information Text}

Section S1. Derivation of xenon host molecule concentration expression

Section S2. Measurement of the binding constant of $\mathrm{CrA}-(\mathrm{COOH})_{6}$

Section S3. Analysis of the standard deviation of concentration determination

Figure $\mathrm{S} 1$. The structure of $\mathrm{CrA}-(\mathrm{COOH})_{6}$.

Figure S2. The CEIT pulse sequence diagram.

Figure S3. $2.5 \mu \mathrm{M} \mathrm{CrA}-(\mathrm{COOH})_{6}{ }^{129} \mathrm{Xe}$ NMR spectroscopy.

Figure S4. The local CEIT z-spectra achieved with different experimental settings.

Figure S5. The exchange rate fitting of $2.5 \mu \mathrm{M} \mathrm{CrA}-(\mathrm{COOH})_{6}$ at $297 \mathrm{~K}$ and $65.7 \mathrm{psi}$.

Figure S6. DLS data showing size distribution by number in aqueous solution at $298 \mathrm{~K}$ of 100.0

$\mu \mathrm{M}$ CrA $-(\mathrm{COOH})_{6}$ and $10.5 \mu \mathrm{M} \mathrm{CrA}-(\mathrm{COOH})_{6}$.

Figure S7. The comparison of the measured $\mathrm{CrA}-(\mathrm{COOH})_{6}$ concentrations and prepared CrA-

$(\mathrm{COOH})_{6}$ concentrations ranges from $10.5 \mathrm{nM}$ to $10.5 \mu \mathrm{M}$.

Figure S8. The CEIT effect of sample S1-S3 under variable unit repeat numbers.

SI References 


\section{Experimental Description}

Sample preparation. The cryptophane-A derivative $\operatorname{CrA}-(\mathrm{COOH})_{6}$ was purchased from a commercial supplier and used without purification. The structure of $\mathrm{CrA}-(\mathrm{COOH})_{6}$ (Figure $\mathrm{S} 1$ ) is shown in the supporting information. As a stock solution, $1.22 \mathrm{mg} \mathrm{CrA}-(\mathrm{COOH})_{6}$ was dissolved in $10 \mathrm{~mL}$ deionized water, and the $\mathrm{pH}$ was adjusted through addition of $\mathrm{NaOH}$ solution $(1 \mathrm{M})$. The solution was then diluted to different concentration levels $(10.5 \mathrm{nM}, 105.0 \mathrm{nM}, 1.05 \mu \mathrm{M}, 10.5 \mu \mathrm{M}$, $100.0 \mu \mathrm{M}, 2.5 \mu \mathrm{M}, 4.9 \mu \mathrm{M}, 7.2 \mu \mathrm{M}$ and $9.3 \mu \mathrm{M})$ for ${ }^{129}$ Xe NMR experiments.

Hyperpolarized ${ }^{129} \mathrm{Xe}$ NMR. Hyperpolarized ${ }^{129} \mathrm{Xe}$ was generated with the spin exchange optical pumping technique using a commercial hyperpolarizer system (verlmagin Healthcare, Wuhan, China). A xenon gas mixture consisting of $2 \% \mathrm{Xe}$ (natural abundance), $10 \% \mathrm{~N}_{2}$, and $88 \% \mathrm{He}$ was used. The temperature in the pumping cell was $418 \mathrm{~K}$, and the gas mixture pressure was $65.7 \mathrm{psi}$. The average ${ }^{129} \mathrm{Xe}$ spin polarization was approximately $10 \%$.

All NMR experiments were conducted on a $400 \mathrm{MHz}$ wide-bore NMR spectrometer (Bruker Avance, Ettlingen, Germany) using a $10 \mathrm{~mm}$ BBO probe. A $10 \mathrm{~mm}$ tube containing the test sample was placed in magnet with the temperature controlled at $297 \mathrm{~K}$. The sample was directly bubbled for 20 $\mathrm{s}$ at a flow rate of 0.12 standard liters per minute, which was followed by a $3 \mathrm{~s}$ delay to allow the sample to remain stable before acquisition.

The signals were referenced to the signal of free xenon in solution ( $0 \mathrm{ppm})$.

\section{Supplementary Information Text}

\section{Section S1. Derivation of xenon host molecule concentration expression}

The dynamics of magnetization in the free $\mathrm{Xe}$ pool, $\mathrm{M}_{\mathrm{Xe}}(\mathrm{t})$, and in the host molecule bound $\mathrm{Xe}$, $\mathrm{M}_{\mathrm{CXe}}(\mathrm{t})$, was described during the periods of delay time $\mathrm{t}$ by Bloch-McConnell equations restricted to the following: 1

$$
\begin{aligned}
\frac{d M_{X e}(t)}{d t} & =M_{X e}^{0} R_{1 X e}-\left(k_{\text {on }}+R_{1 X e}\right) M_{X e}(t)+k_{\text {off }} M_{C X e}(t) \\
\frac{d M_{C X e}(t)}{d t} & =M_{C X e}^{0} R_{1 C X e}+k_{o n} M_{X e}(t)-\left(k_{\text {off }}+R_{1 C X e}\right) M_{C X e}(t)
\end{aligned}
$$

The thermal equilibrium magnetizations were denoted as $\mathrm{M}_{\mathrm{Xe}}^{0}$ and $\mathrm{M}_{\mathrm{CXe}}^{0}$. The longitudinal relaxation rates were denoted as $\mathrm{R}_{1 \mathrm{Xe}}$ and $\mathrm{R}_{1 \mathrm{CXe}}$. The inverse lifetimes of free $\mathrm{Xe}$ and $\mathrm{Xe}$ in the host molecule bound pools were denoted as $\mathrm{k}_{\text {off }}$ and $\mathrm{k}_{\mathrm{on}}$, respectively. To obtain a simple expression, some reasonable assumptions were made. First, the constant terms $\mathrm{M}_{\mathrm{Xe}}^{0}$ and $\mathrm{M}_{\mathrm{CXe}}^{0}$ were negligible because of the very low thermal polarization compared with the hyperpolarized magnetizations $\mathrm{M}_{\mathrm{Xe}}(\mathrm{t})$ and $\mathrm{M}_{\mathrm{CXe}}(\mathrm{t})$ prevailing during the experiments. Second, the longitudinal relaxation rate for xenon bound to host molecule, $\mathrm{R}_{1 \mathrm{CXe}}$, was less than a tenth of 1 hertz and can be completely ignored in (A2) in comparison with the dissociation rate $\mathrm{k}_{\text {off }}$, which was of the order of tens of hertz in aqueous solution and even higher in organic solvents. Disregarding $R_{1 X e}$ against $k_{o n}$ in (A1) was not generally justified because of the smallness of $\mathrm{k}_{\mathrm{on}}$, particularly at low host concentrations. The systems (A1) and (A2) became the following:

$$
\begin{aligned}
& \frac{d M_{X e}(t)}{d t}=-\left(k_{o n}+R_{1 X e}\right) M_{X e}(t)+k_{\text {off }} M_{C X e}(t) \\
& \frac{d M_{C X e}(t)}{d t}=k_{o n} M_{X e}(t)-k_{\text {off }} M_{C X e}(t)
\end{aligned}
$$

The differential equations (A1') and (A2') were solved.

$$
\begin{aligned}
& M_{X e}(t)=-C_{2} e^{\left(t \lambda_{1}\right)} \frac{d-\lambda_{1}}{c}-C_{1} e^{\left(t \lambda_{2}\right)} \frac{d-\lambda_{2}}{c} \\
& M_{C X e}(t)=C_{2} e^{\left(t \lambda_{1}\right)}+C_{1} e^{\left(t \lambda_{2}\right)}
\end{aligned}
$$

In which, $\mathrm{c}=\mathrm{k}_{\mathrm{on}}, \mathrm{d}=-\mathrm{k}_{\mathrm{off}}, \mathrm{C}_{1}$ and $\mathrm{C}_{2}$ were constants.

$$
\begin{aligned}
& \lambda_{1}=\frac{-k_{\text {on }}-R_{1 X e}-k_{\text {off }}+\sqrt{\left(k_{\text {off }}-k_{\text {on }}-R_{1 X e}\right)^{2}+4 k_{\text {off }} k_{\text {on }}}}{2} \\
& \lambda_{2}=\frac{-k_{\text {on }}-R_{1 X e}-k_{\text {off }}-\sqrt{\left(k_{\text {off }}-k_{\text {on }}-R_{1 X e}\right)^{2}+4 k_{\text {off }} k_{\text {on }}}}{2}
\end{aligned}
$$

Considering that $\mathrm{k}_{\text {off }} \gg \mathrm{k}_{\text {on }}>0$ and $\mathrm{k}_{\text {off }} \gg \mathrm{R}_{1 \text { Xe }}>0$, we obtained $\left(\mathrm{k}_{\text {off }}-\mathrm{R}_{1 \text { Xe }}+\mathrm{k}_{\text {on }}\right)^{2} \gg 4 \mathrm{k}_{\text {on }} \mathrm{R}_{1 \mathrm{Xe}}$, and the expressions of $\lambda_{1}$ and $\lambda_{2}$ were simplified into the following: 


$$
\begin{aligned}
& \lambda_{1}=\frac{-\left(\mathrm{k}_{\mathrm{on}}+\mathrm{R}_{1 \mathrm{Xe}}+\mathrm{k}_{\mathrm{off}}\right)+\sqrt{\left(\mathrm{k}_{\mathrm{off}}-\mathrm{R}_{1 \mathrm{Xe}}-\mathrm{k}_{\mathrm{on}}\right)^{2}+4 \mathrm{k}_{\mathrm{on}} \mathrm{k}_{\mathrm{off}}}}{2} \\
& =\frac{-\left(\mathrm{k}_{\text {on }}+\mathrm{R}_{1 \mathrm{Xe}}+\mathrm{k}_{\text {off }}\right)+\sqrt{\left(\mathrm{k}_{\text {off }}-\mathrm{R}_{1 \mathrm{Xe}}+\mathrm{k}_{\text {on }}\right)^{2}+4 \mathrm{k}_{\text {on }} \mathrm{R}_{1 \mathrm{Xe}}}}{2} \\
& \approx \frac{-\left(\mathrm{k}_{\mathrm{on}}+\mathrm{R}_{1 \mathrm{Xe}}+\mathrm{k}_{\mathrm{off}}\right)+\sqrt{\left(\mathrm{k}_{\mathrm{off}}-\mathrm{R}_{1 \mathrm{Xe}}+\mathrm{k}_{\mathrm{on}}\right)^{2}}}{2} \\
& \lambda_{2}=\frac{-\left(\mathrm{k}_{\mathrm{on}}+\mathrm{R}_{1 \mathrm{Xe}}+\mathrm{k}_{\mathrm{of} f}\right)-\sqrt{\left(\mathrm{k}_{\mathrm{off}}-\mathrm{R}_{1 \mathrm{Xe}}-\mathrm{k}_{\mathrm{on}}\right)^{2}+4 \mathrm{k}_{\mathrm{on}} \mathrm{k}_{\mathrm{off}}}}{2} \\
& =\frac{-\left(\mathrm{k}_{\mathrm{on}}+\mathrm{R}_{1 \mathrm{Xe}}+\mathrm{k}_{\mathrm{of} f}\right)-\sqrt{\left(\mathrm{k}_{\mathrm{off}}-\mathrm{R}_{1 \mathrm{Xe}}+\mathrm{k}_{\mathrm{on}}\right)^{2}+4 \mathrm{k}_{\mathrm{on}} \mathrm{R}_{1 \mathrm{Xe}}}}{2} \\
& \approx \frac{-\left(\mathrm{k}_{\mathrm{on}}+\mathrm{R}_{1 \mathrm{Xe}}+\mathrm{k}_{\mathrm{off}}\right)-\sqrt{\left(\mathrm{k}_{\mathrm{off}}-\mathrm{R}_{1 \mathrm{Xe}}+\mathrm{k}_{\mathrm{on}}\right)^{2}}}{2 \approx-\left(\mathrm{k}_{\mathrm{on}}+\mathrm{k}_{\mathrm{off}}\right)}
\end{aligned}
$$

Before the free chemical exchange process started, $t=0$, and both $\mathrm{e}^{\left(\mathrm{t} \lambda_{1}\right)}$ and $\mathrm{e}^{\left(\mathrm{t} \lambda_{2}\right)}$ equaled 1 . $\mathrm{M}_{\mathrm{Xe}}(0)$ and $\mathrm{M}_{\mathrm{CXe}}(0)$ were the magnetizations of free Xe and host molecule bound $\mathrm{Xe}$ at $\mathrm{t}=0$. Thus, equation (A3) and (A4) changed into the following:

$$
\begin{aligned}
& \mathrm{M}_{\mathrm{Xe}}(0)=-\mathrm{C}_{2} \frac{-\mathrm{k}_{\text {off }}-\lambda_{1}}{\mathrm{k}_{\mathrm{on}}}-\mathrm{C}_{1} \frac{-\mathrm{k}_{\text {off }}-\lambda_{2}}{\mathrm{k}_{\mathrm{on}}} \\
& \mathrm{M}_{\mathrm{CXe}}(0)=\mathrm{C}_{2}+\mathrm{C}_{1}
\end{aligned}
$$

Then, we achieved the expressions of constant $\mathrm{C}_{1}$ and $\mathrm{C}_{2}$, as follows:

$$
\begin{aligned}
C_{1} & =\frac{M_{X e}(0) k_{\text {on }}-M_{C_{X e}}(0)\left(k_{\text {off }}-R_{1 X e}\right)}{R_{1 X}-k_{\text {on }}-k_{\text {off }}} \\
C_{2} & =\frac{M_{X e}(0) k_{\text {on }}+M_{C X e}(0) k_{\text {on }}}{-R_{1 X e}+k_{\text {on }}+k_{\text {off }}}
\end{aligned}
$$

As shown in Figure S2, the magnetization of free $\mathrm{Xe}$ and host molecule bound Xe after the $n$th $180^{\circ}$ inversion pulse were denoted as $\mathrm{M}_{\mathrm{Xe}}^{(\mathrm{n})}(0)$ and $\mathrm{M}_{\mathrm{CXe}}^{(\mathrm{n})}(0)$ respectively. The magnetizations of free Xe and host molecule bound $\mathrm{Xe}$ after delay time $\mathrm{t}$ for free exchange were respectively denoted as $\mathrm{M}_{\mathrm{Xe}}^{(\mathrm{n})}(\mathrm{t})$ and $\mathrm{M}_{\mathrm{CXe}}^{(\mathrm{n})}(\mathrm{t})$. Furthermore, we defined a parameter $\beta^{(n-1)}$, and $\mathrm{M}_{\mathrm{CXe}}^{(\mathrm{n})}(0)=-\beta^{(n-1)} \mathrm{fr} \mathrm{M}_{\mathrm{Xe}}^{(\mathrm{n})}(0)$, fr was the ratio between host molecule bound $X e$ and free Xe. The expressions of $M_{X e}^{(n)}(t)$ and $\mathrm{M}_{\mathrm{CXe}}^{(\mathrm{n})}(\mathrm{t})$ in equation (A3) and (A4) became the following:

$$
\begin{aligned}
& M_{X e}^{(n)}(t)=-C_{2} e^{\left(t \lambda_{1}\right)} \frac{d-\lambda_{1}}{c}-C_{1} e^{\left(t \lambda_{2}\right)} \frac{d-\lambda_{2}}{c} \\
& \approx-\frac{\mathrm{M}_{\mathrm{Xe}}^{(\mathrm{n})}(0) \mathrm{k}_{\text {on }}+\mathrm{M}_{\mathrm{CXe}}^{(\mathrm{n})}(0) \mathrm{k}_{\text {on }}}{-\mathrm{R}_{1 \mathrm{Xe}}+\mathrm{k}_{\text {on }}+\mathrm{k}_{\text {off }}} \mathrm{e}^{\left(\mathrm{t}\left(-\mathrm{R}_{1 \mathrm{Xe}}\right)\right)} \frac{-\mathrm{k}_{\text {off }}-\left(-\mathrm{R}_{1 \mathrm{Xe}}\right)}{\mathrm{k}_{\text {on }}} \\
& -\frac{\mathrm{M}_{\mathrm{Xe}}^{(\mathrm{n})}(0) \mathrm{k}_{\text {on }}-\mathrm{M}_{\mathrm{CXe}}^{(\mathrm{n})}(0)\left(\mathrm{k}_{\text {off }}-\mathrm{R}_{1 \mathrm{Xe}}\right)}{\mathrm{R}_{1 \mathrm{Xe}}-\mathrm{k}_{\text {on }}-\mathrm{k}_{\text {off }}} \mathrm{e}^{\left(\mathrm{t}\left(-\left(\mathrm{k}_{\text {on }}+\mathrm{k}_{\text {off }}\right)\right)\right.} \frac{-\mathrm{k}_{\text {off }}-\left(-\left(\mathrm{k}_{\text {on }}+\mathrm{k}_{\text {off }}\right)\right)}{\mathrm{k}_{\text {on }}} \\
& \approx-\frac{\mathrm{M}_{\mathrm{Xe}}^{(\mathrm{n})}(0) \mathrm{k}_{\mathrm{on}}+\left(-\beta^{(n-1)} \mathrm{fr} \mathrm{M}_{\mathrm{Xe}}^{(\mathrm{n})}(0)\right) \mathrm{k}_{\mathrm{on}}}{-\mathrm{R}_{1 \mathrm{Xe}}+\mathrm{k}_{\mathrm{on}}+\mathrm{k}_{\mathrm{off}}} \mathrm{e}^{\left(-\mathrm{tR}_{1 \mathrm{Xe}}\right)} \frac{-\mathrm{k}_{\mathrm{off}}+\mathrm{R}_{1 \mathrm{Xe}}}{\mathrm{k}_{\mathrm{on}}} \\
& -\frac{\mathrm{M}_{\mathrm{Xe}}^{(\mathrm{n})}(0) \mathrm{k}_{\mathrm{on}}-\left(-\beta^{(n-1)} \mathrm{fr} \mathrm{M}_{\mathrm{Xe}}^{(\mathrm{n})}(0)\right)\left(\mathrm{k}_{\text {off }}-\mathrm{R}_{1 \mathrm{Xe}}\right)}{\mathrm{R}_{1 \mathrm{Xe}}-\mathrm{k}_{\mathrm{on}}-\mathrm{k}_{\text {off }}} \mathrm{e}^{\left(-\mathrm{t}\left(\mathrm{k}_{\mathrm{on}}+\mathrm{k}_{\text {off }}\right)\right.} \frac{\mathrm{k}_{\mathrm{on}}}{\mathrm{k}_{\text {on }}} \\
& \approx-\frac{\mathrm{M}_{\mathrm{Xe}}^{(\mathrm{n})}(0) \mathrm{fr} \mathrm{k}_{\mathrm{off}}-\beta^{(n-1)} \mathrm{fr} \mathrm{M}_{\mathrm{Xe}}^{(\mathrm{n})}(0) \mathrm{fr} \mathrm{k}_{\text {off }}}{-\mathrm{R}_{1 \mathrm{Xe}}+\mathrm{fr} \mathrm{k}_{\text {off }}+\mathrm{k}_{\text {off }}} \mathrm{e}^{\left(-\mathrm{tR}_{1 \mathrm{Xe}}\right)} \frac{-\mathrm{k}_{\text {off }}+\mathrm{R}_{1 \mathrm{Xe}}}{\text { fr } \mathrm{k}_{\text {off }}} \\
& -\frac{\mathrm{M}_{\mathrm{Xe}}^{(\mathrm{n})}(0) \mathrm{fr} \mathrm{k}_{\text {off }}+\beta^{(n-1)} \mathrm{fr} \mathrm{M}_{\mathrm{Xe}}^{(\mathrm{n})}(0)\left(\mathrm{k}_{\text {off }}-\mathrm{R}_{1 \mathrm{Xe}}\right)}{\mathrm{R}_{1 \mathrm{Xe}}-\mathrm{fr} \mathrm{k}_{\text {off }}-\mathrm{k}_{\mathrm{off}}} \mathrm{e}^{\left(-\mathrm{t}\left(\mathrm{k}_{\mathrm{on}}+\mathrm{k}_{\text {off }}\right)\right)} \\
& \approx-\frac{\mathrm{M}_{\mathrm{Xe}}^{(\mathrm{n})}(0)-\beta^{(n-1)} \mathrm{fr}_{\mathrm{Xe}}^{(\mathrm{n})}(0)}{-\mathrm{R}_{1 \mathrm{Xe}}+\mathrm{fr}_{\mathrm{off}}+\mathrm{k}_{\mathrm{off}}} \mathrm{e}^{\left(-\mathrm{tR}_{1 \mathrm{Xe}}\right)}\left(-\mathrm{k}_{\mathrm{off}}+\mathrm{R}_{1 \mathrm{Xe}}\right) \\
& -\frac{\mathrm{M}_{\mathrm{Xe}}^{(\mathrm{n})}(0) \mathrm{fr} \mathrm{k}_{\mathrm{off}}+\beta^{(n-1)} \mathrm{fr} \mathrm{M}_{\mathrm{Xe}}^{(\mathrm{n})}(0)\left(\mathrm{k}_{\text {off }}-\mathrm{R}_{1 \mathrm{Xe}}\right)}{\mathrm{R}_{1 \mathrm{Xe}}-\mathrm{frk}_{\mathrm{off}}-\mathrm{k}_{\mathrm{off}}} \mathrm{e}^{\left(-\mathrm{t}\left(\mathrm{k}_{\mathrm{on}}+\mathrm{k}_{\text {off }}\right)\right)}
\end{aligned}
$$




$$
\begin{aligned}
& \approx \frac{\mathrm{M}_{\mathrm{Xe}}^{(\mathrm{n})}(0)-\beta^{(n-1)} \mathrm{fr} \mathrm{M}_{\mathrm{Xe}}^{(\mathrm{n})}(0)}{-\mathrm{R}_{1 \mathrm{Xe}}+\text { fr } \mathrm{k}_{\mathrm{off}}+\mathrm{k}_{\mathrm{off}}} \mathrm{e}^{\left(-\mathrm{tR}_{1 \mathrm{Xe}}\right)}\left(\mathrm{k}_{\mathrm{off}}-\mathrm{R}_{1 \mathrm{Xe}}\right) \\
& +\frac{\mathrm{M}_{\mathrm{Xe}}^{(\mathrm{n})}(0) \mathrm{fr} \mathrm{k}_{\mathrm{off}}+\beta^{(n-1)} \mathrm{fr} \mathrm{M}_{\mathrm{Xe}}^{(\mathrm{n})}(0)\left(\mathrm{k}_{\text {off }}-\mathrm{R}_{1 \mathrm{Xe}}\right)}{-\mathrm{R}_{1 \mathrm{Xe}}+\text { fr } \mathrm{k}_{\text {off }}+\mathrm{k}_{\text {off }}} \mathrm{e}^{\left(-\mathrm{t}\left(\mathrm{k}_{\mathrm{on}}+\mathrm{k}_{\text {off }}\right)\right)} \\
& \approx \mathrm{M}_{\mathrm{Xe}}^{(\mathrm{n})}(0) \frac{1-\beta^{(n-1)} \mathrm{fr}}{-\mathrm{R}_{1 \mathrm{Xe}}+\mathrm{fr}_{\mathrm{off}}+\mathrm{k}_{\text {off }}} \mathrm{e}^{\left(-\mathrm{tR}_{1 \mathrm{Xe}}\right)}\left(\mathrm{k}_{\mathrm{off}}-\mathrm{R}_{1 \mathrm{Xe}}\right)+\mathrm{M}_{\mathrm{Xe}}^{(\mathrm{n})}(0) \frac{\mathrm{fr}_{\mathrm{off}}+\beta^{(n-1)} \mathrm{fr}_{\left(\mathrm{k}_{\text {off }}-\mathrm{R}_{1 \mathrm{Xe}}\right)}}{-\mathrm{R}_{1 \mathrm{Xe}}+\mathrm{fr} \mathrm{k}_{\mathrm{off}}+\mathrm{k}_{\mathrm{off}}} \mathrm{e}^{\left(-\mathrm{t}\left(\mathrm{k}_{\mathrm{on}}+\mathrm{k}_{\text {off }}\right)\right)}
\end{aligned}
$$

$$
\begin{aligned}
& \mathrm{M}_{\mathrm{CXe}}^{(\mathrm{n})}(\mathrm{t})=\mathrm{C}_{2} \mathrm{e}^{\left(\mathrm{t} \lambda_{1}\right)}+\mathrm{C}_{1} \mathrm{e}^{\left(\mathrm{t} \lambda_{2}\right)} \\
& \approx \frac{\mathrm{M}_{\mathrm{Xe}}^{(\mathrm{n})}(0) \mathrm{k}_{\text {on }}+\mathrm{M}_{\mathrm{CXe}}^{(\mathrm{n})}(0) \mathrm{k}_{\text {on }}}{-\mathrm{R}_{1 \mathrm{Xe}}+\mathrm{k}_{\text {on }}+\mathrm{k}_{\text {off }}} \mathrm{e}^{\left(\mathrm{t}\left(-\mathrm{R}_{1 \mathrm{Xe}}\right)\right)}+\frac{\mathrm{M}_{\mathrm{Xe}}^{(\mathrm{n})}(0) \mathrm{k}_{\text {on }}-\mathrm{M}_{\mathrm{CXe}}^{(\mathrm{n})}(0)\left(\mathrm{k}_{\text {off }}-\mathrm{R}_{1 \mathrm{Xe}}\right)}{\mathrm{R}_{1 \mathrm{Xe}}-\mathrm{k}_{\text {on }}-\mathrm{k}_{\text {off }}} \mathrm{e}^{\left(\mathrm{t}\left(-\left(\mathrm{k}_{\text {on }}+\mathrm{k}_{\text {off }}\right)\right)\right)} \\
& \approx \frac{\mathrm{M}_{\mathrm{Xe}}^{(\mathrm{n})}(0) \mathrm{fr} \mathrm{k}_{\mathrm{off}}+\left(-\beta^{(n-1)} \mathrm{fr} \mathrm{M}_{\mathrm{Xe}}^{(\mathrm{n})}(0)\right) \mathrm{fr} \mathrm{k}_{\text {off }}}{-\mathrm{R}_{1 \mathrm{Xe}}+\mathrm{fr}_{\mathrm{off}}+\mathrm{k}_{\text {off }}} \mathrm{e}^{\left(\mathrm{t}\left(-\mathrm{R}_{1 \mathrm{Xe}}\right)\right)} \\
& +\frac{\mathrm{M}_{\mathrm{Xe}}^{(\mathrm{n})}(0) f r \mathrm{k}_{\text {off }}-\left(-\beta^{(n-1)} \mathrm{fr} \mathrm{M}_{\mathrm{Xe}}^{(\mathrm{n})}(0)\right)\left(\mathrm{k}_{\text {off }}-\mathrm{R}_{1 \mathrm{Xe}}\right)}{\mathrm{R}_{1 \mathrm{Xe}}-\mathrm{fr}_{\text {off }}-\mathrm{k}_{\text {off }}} \mathrm{e}^{\left(\mathrm{t}\left(-\left(\mathrm{k}_{\text {on }}+\mathrm{k}_{\text {off }}\right)\right)\right.} \\
& \approx \mathrm{M}_{\mathrm{Xe}}^{(\mathrm{n})}(0) \mathrm{e}^{\left(\mathrm{t}\left(-\mathrm{R}_{1 \mathrm{Xe}}\right)\right)} \mathrm{fr}\left(\frac{\mathrm{k}_{\text {off }}-\beta^{(n-1)} \text { fr } \mathrm{k}_{\text {off }}}{-\mathrm{R}_{1 \mathrm{Xe}}+\mathrm{fr}_{\text {off }}+\mathrm{k}_{\text {off }}}+\frac{\mathrm{k}_{\text {off }}+\beta^{(n-1)}\left(\mathrm{k}_{\text {off }}-\mathrm{R}_{1 \mathrm{Xe}}\right)}{\mathrm{R}_{1 \mathrm{Xe}}-\mathrm{fr} \mathrm{k}_{\text {off }}-\mathrm{k}_{\text {off }}} \mathrm{e}^{\left(\mathrm{t}\left(\mathrm{R}_{1 \mathrm{Xe}}-\left(\mathrm{k}_{\text {on }}+\mathrm{k}_{\text {off }}\right)\right)\right)}\right)
\end{aligned}
$$

Considering that $\mathrm{k}_{\text {off }} \gg \mathrm{R}_{1 \mathrm{Xe}}>0$, we achieved $\left(\mathrm{k}_{\text {off }}-\mathrm{R}_{1 \text { Xe }}\right) \approx \mathrm{k}_{\text {off }}$ and fr $\mathrm{k}_{\text {off }}+\mathrm{k}_{\text {off }}-\mathrm{R}_{1 \text { Xe }} \approx(1+$ fr) $\mathrm{k}_{\text {off. }}$. In addition, $\frac{1}{\text { fr }+1} \approx 1$, because $1 \gg$ fr. The expression (A9) and (A10) was further simplified into the following:

$$
\begin{aligned}
& \mathrm{M}_{\mathrm{Xe}}^{(\mathrm{n})}(\mathrm{t}) \approx \mathrm{M}_{\mathrm{Xe}}^{(\mathrm{n})}(0) \frac{1-\beta^{(n-1)} \mathrm{fr}}{(1+\mathrm{fr}) \mathrm{k}_{\text {off }}} \mathrm{e}^{\left(-\mathrm{tR}_{1 \mathrm{Xe}}\right)} \mathrm{k}_{\text {off }}+\mathrm{M}_{\mathrm{Xe}}^{(\mathrm{n})}(0) \frac{\text { fr } \mathrm{k}_{\text {off }}+\beta^{(n-1)} \mathrm{fr} \mathrm{k}_{\text {off }}}{(1+\mathrm{fr}) \mathrm{k}_{\text {off }}} \mathrm{e}^{\left(-\mathrm{t}\left(\mathrm{k}_{\text {on }}+\mathrm{k}_{\text {off }}\right)\right)} \\
& \approx \mathrm{M}_{\mathrm{Xe}}^{(\mathrm{n})}(0) \frac{1-\beta^{(n-1)} \mathrm{fr}}{(1+\mathrm{fr})} \mathrm{e}^{\left(-\mathrm{tR}_{1 \mathrm{Xe}}\right)}+\mathrm{M}_{\mathrm{Xe}}^{(\mathrm{n})}(0) \frac{\mathrm{fr}+\beta^{(n-1)} \mathrm{fr}}{(1+\mathrm{fr})} \mathrm{e}^{\left(-\mathrm{t}\left(\mathrm{k}_{\mathrm{on}}+\mathrm{k}_{\mathrm{off}}\right)\right)} \\
& \approx \mathrm{M}_{\mathrm{Xe}}^{(\mathrm{n})}(0) \mathrm{e}^{\left(-\mathrm{tR}_{1 \mathrm{Xe}}\right)}\left(\frac{1+\mathrm{fr}-\mathrm{fr}-\beta^{(n-1)} \mathrm{fr}}{(1+\mathrm{fr})}+\frac{\left(1+\beta^{(n-1)}\right) \mathrm{fr}}{(1+\mathrm{fr})} \mathrm{e}^{\left(-\mathrm{t}\left(\mathrm{k}_{\mathrm{on}}+\mathrm{k}_{\mathrm{off}}-\mathrm{R}_{1 \mathrm{Xe}}\right)\right)}\right) \\
& \approx \mathrm{M}_{\mathrm{Xe}}^{(\mathrm{n})}(0) \mathrm{e}^{\left(-\mathrm{tR} \mathrm{R}_{1 \mathrm{Xe}}\right)}\left(1-\left(1+\beta^{(n-1)}\right) \mathrm{fr}+\left(1+\beta^{(n-1)}\right) \mathrm{fr}^{\left(-\mathrm{t}\left(\mathrm{k}_{\mathrm{on}}+\mathrm{k}_{\mathrm{off}}-\mathrm{R}_{1 \mathrm{Xe}}\right)\right)}\right) \\
& \approx \mathrm{M}_{\mathrm{Xe}}^{(\mathrm{n})}(0) \mathrm{e}^{\left(-\mathrm{tR}_{1 \mathrm{Xe}}\right)}\left(1-\left(1+\beta^{(n-1)}\right) \operatorname{fr}\left(1-\mathrm{e}^{\left(-\mathrm{t}\left(\mathrm{k}_{\mathrm{on}}+\mathrm{k}_{\text {off }}-\mathrm{R}_{1 \mathrm{Xe}}\right)\right)}\right)\right) \\
& \mathrm{M}_{\mathrm{CXe}}^{(\mathrm{n})}(\mathrm{t}) \approx \mathrm{M}_{\mathrm{Xe}}^{(\mathrm{n})}(0) \mathrm{e}^{\left(\mathrm{t}\left(-\mathrm{R}_{1 \mathrm{Xe}}\right)\right)} \mathrm{fr}\left(\frac{\left(1-\beta^{(n-1)} \mathrm{fr}\right) \mathrm{k}_{\text {off }}}{(1+\mathrm{fr}) \mathrm{k}_{\mathrm{off}}}+\frac{\left(1+\beta^{(n-1)}\right) \mathrm{k}_{\text {off }}}{-(1+\mathrm{fr}) \mathrm{k}_{\mathrm{off}}} \mathrm{e}^{\left(\mathrm{t}\left(\mathrm{R}_{1 \mathrm{Xe}^{-}}-\left(\mathrm{k}_{\text {on }}+\mathrm{k}_{\text {off }}\right)\right)\right)}\right) \\
& \approx \mathrm{M}_{\mathrm{Xe}}^{(\mathrm{n})}(0) \mathrm{e}^{\left(\mathrm{t}\left(-\mathrm{R}_{1 \mathrm{Xe}}\right)\right)} \mathrm{fr}\left(\frac{\left(1-\beta^{(n-1)} \mathrm{fr}\right)}{(1+\mathrm{fr})}-\frac{\left(1+\beta^{(n-1)}\right)}{(1+\mathrm{fr})} \mathrm{e}^{\left(\mathrm{t}\left(\mathrm{R}_{1 \mathrm{Xe}}-\left(\mathrm{k}_{\mathrm{on}}+\mathrm{k}_{\mathrm{off}}\right)\right)\right)}\right) \\
& \approx \mathrm{M}_{\mathrm{Xe}}^{(\mathrm{n})}(0) \mathrm{e}^{\left(\mathrm{t}\left(-\mathrm{R}_{1 \mathrm{Xe}}\right)\right)} \mathrm{fr}\left(\frac{(1+\mathrm{fr})-\left(1+\beta^{(n-1)}\right) \mathrm{fr}-\left(1+\beta^{(n-1)}\right) \mathrm{e}^{\left(\mathrm{t}\left(\mathrm{R}_{1 \mathrm{Xe}}-\left(\mathrm{k}_{\mathrm{on}}+\mathrm{k}_{\mathrm{off}}\right)\right)\right)}}{(1+\mathrm{fr})}\right) \\
& \approx \mathrm{M}_{\mathrm{Xe}}^{(\mathrm{n})}(0) \mathrm{e}^{\left(\mathrm{t}\left(-\mathrm{R}_{1 \mathrm{Xe}}\right)\right)} \mathrm{fr}\left(1-\left(1+\beta^{(n-1)}\right)\left(\mathrm{fr}+\mathrm{e}^{\left(\mathrm{t}\left(\mathrm{R}_{1 \mathrm{Xe}}-\left(\mathrm{k}_{\mathrm{on}}+\mathrm{k}_{\mathrm{off}}\right)\right)\right)}\right)\right)
\end{aligned}
$$

We defined another parameter $\alpha^{(\mathrm{n})}$ as follows:

$$
\begin{aligned}
& \alpha^{(\mathrm{n})}=\frac{\mathrm{M}_{\mathrm{CXe}}^{(\mathrm{n})}(\mathrm{t})}{\operatorname{fr}_{\mathrm{Xe}}^{(\mathrm{n})}(\mathrm{t})} \approx \frac{\mathrm{M}_{\mathrm{Xe}}^{(\mathrm{n})}(0) \mathrm{fr}^{-\mathrm{R}_{1 X \mathrm{e}} \mathrm{t}}\left(1-\left(1+\beta^{(\mathrm{n}-1)}\right)\left(\mathrm{fr}+\mathrm{e}^{\mathrm{t}\left(\mathrm{R}_{1 \mathrm{Xe}}-\mathrm{k}_{\mathrm{on}}-\mathrm{k}_{\mathrm{off}}\right)}\right)\right)}{\operatorname{fr}_{\mathrm{Xe}}^{(\mathrm{n})}(0) \mathrm{e}^{-\mathrm{R}_{1 \mathrm{Xe}} \mathrm{t}}\left(1-\left(1+\beta^{(n-1)}\right) \mathrm{fr}\left(1-\mathrm{e}^{\mathrm{t}\left(\mathrm{R}_{1 \mathrm{Xe}}-\mathrm{k}_{\mathrm{on}}-\mathrm{k}_{\mathrm{off}}\right)}\right)\right)}
\end{aligned}
$$

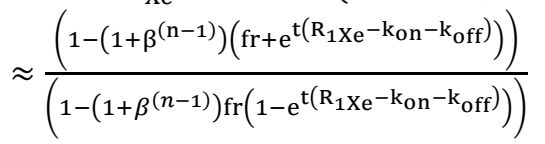

In an off-resonance experiment, $\quad \mathrm{M}_{\mathrm{Xe}}^{(\mathrm{n})}(0)=\mathrm{M}_{\mathrm{Xe}}^{(\mathrm{n}-1)}(\mathrm{t}) \mathrm{e}^{-\mathrm{R}_{1 \times \mathrm{e}} \mathrm{t}_{\mathrm{p}}} \quad$ and $\quad \mathrm{M}_{\mathrm{CXe}}^{(\mathrm{n})}(0)=$ $\alpha^{(\mathrm{n})} \mathrm{fr}_{\mathrm{Xe}}^{(\mathrm{n}-1)}(\mathrm{t}) \mathrm{e}^{-\mathrm{R}_{1} \mathrm{Xe}_{\mathrm{p}}}=-\beta^{(\mathrm{n})} \mathrm{fr} \mathrm{M}_{\mathrm{Xe}}^{(\mathrm{n}-1)}(\mathrm{t}) \mathrm{e}^{-\mathrm{R}_{1 \times \mathrm{Xe}} \mathrm{t}_{\mathrm{p}}}$. Thus, $\beta^{(\mathrm{n})}=-\alpha^{(\mathrm{n})}$. We obtained an iterative expression of free Xe magnetization that was related to the $n$th $180^{\circ}$ inversion pulse-delay to the $(n-1)$ th under arbitrarily delay time $t$, as follows: 


$$
\begin{aligned}
& \mathrm{M}_{\mathrm{Xe}}^{(\mathrm{n})}(\mathrm{t}) \approx \mathrm{M}_{\mathrm{Xe}}^{(\mathrm{n}-1)}(\mathrm{t}) \mathrm{e}^{-\mathrm{R}_{1 \mathrm{Xe}} \mathrm{t}_{\mathrm{p}}} \mathrm{e}^{\left(-\mathrm{tR}_{1 \mathrm{Xe}}\right)}\left(1-\left(1-\alpha^{(\mathrm{n}-1)}\right) \operatorname{fr}\left(1-\mathrm{e}^{\left(-\mathrm{t}\left(\mathrm{k}_{\mathrm{on}}+\mathrm{k}_{\text {off }}-\mathrm{R}_{1 \mathrm{Xe}}\right)\right)}\right)\right)
\end{aligned}
$$

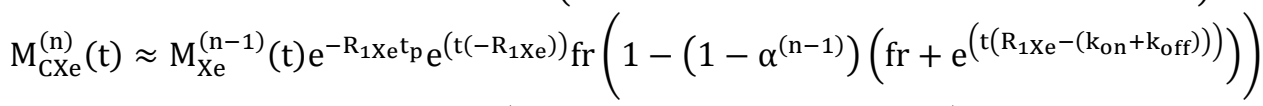

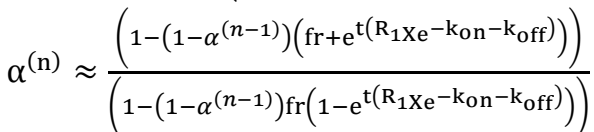

With sufficient delay time for free chemical exchange, we obtained $\mathrm{e}^{\mathrm{t}\left(\mathrm{R}_{1} \mathrm{Xe}-\mathrm{k}_{\mathrm{on}}-\mathrm{k}_{\text {off }}\right)} \approx 0$. Thus, we obtained $\alpha^{(\mathrm{n})} \approx 1$ for arbitrarily $180^{\circ}$ inversion pulse-delay number $\mathrm{n}$. We further $\operatorname{simplified} \mathrm{M}_{\mathrm{Xe}}^{(\mathrm{n})}(t)$ and $\mathrm{M}_{\mathrm{CXe}}^{(\mathrm{n})}(t)$ into the following:

$$
\begin{aligned}
& \mathrm{M}_{\mathrm{Xe}}^{(\mathrm{n})}(\mathrm{t}) \approx \mathrm{M}_{\mathrm{Xe}}^{(\mathrm{n}-1)}(\mathrm{t}) \mathrm{e}^{-\mathrm{R}_{1 X \mathrm{X}} \mathrm{t}_{\mathrm{p}}} \mathrm{e}^{\left(-\mathrm{t}_{1 X \mathrm{Xe}}\right)} \\
& \mathrm{M}_{\mathrm{CXe}}^{(\mathrm{n})}(\mathrm{t}) \approx \mathrm{M}_{\mathrm{Xe}}^{(\mathrm{n}-1)}(\mathrm{t}) \mathrm{e}^{-\mathrm{R}_{1 X e} \mathrm{t}_{\mathrm{p}} \mathrm{e}^{\left(\mathrm{t}\left(-\mathrm{R}_{1 X}\right)\right)} \mathrm{fr}}
\end{aligned}
$$

Then, we obtained the magnetization expression of free $X e$ after the $n$th $180^{\circ}$ inverse pulse-delay unit in an off-resonance experiment, as follows:

$$
\mathrm{M}_{\text {Xe_off }}(\mathrm{t}) \approx \mathrm{M}_{\mathrm{Xe}}^{(0)}(0) \mathrm{e}^{-\mathrm{nR}_{1 \mathrm{Xe}}\left(\mathrm{t}_{\mathrm{p}}+\mathrm{t}\right)}
$$

However, in an on-resonance experiment, $\mathrm{M}_{\mathrm{Xe}}^{(\mathrm{n})}(0)=\mathrm{M}_{\mathrm{Xe}}^{(\mathrm{n}-1)}(\mathrm{t}) \mathrm{e}^{-\mathrm{R}_{1 \mathrm{Xe}} \mathrm{t}_{\mathrm{p}}}$ and $\mathrm{M}_{\mathrm{CXe}}^{(\mathrm{n})}(0)=$ $-\alpha^{(\mathrm{n})} \operatorname{fr~}_{\mathrm{Xe}}^{(\mathrm{n}-1)}(\mathrm{t}) \mathrm{e}^{-\mathrm{R}_{1 \mathrm{Xe}} \mathrm{t}_{\mathrm{p}}}=-\beta^{(\mathrm{n})} \mathrm{fr} \mathrm{M}_{\mathrm{Xe}}^{(\mathrm{n}-1)}(\mathrm{t}) \mathrm{e}^{-\mathrm{R}_{1 \mathrm{Xe}} \mathrm{t}_{\mathrm{p}}}$. Thus, $\beta^{(\mathrm{n})}=\alpha^{(\mathrm{n})}$. We obtained an iterative expression of free Xe magnetization that was related to the $n$th $180^{\circ}$ inversion pulse-delay to the $(n-1)$ th under arbitrarily delay time $t$, as follows:

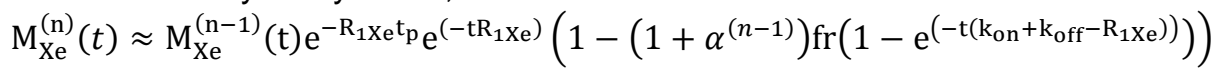

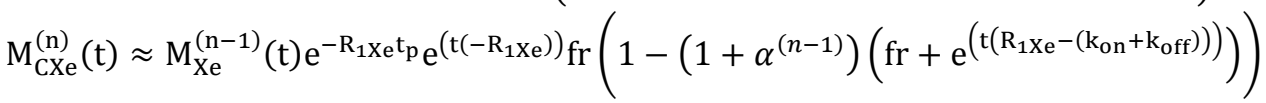

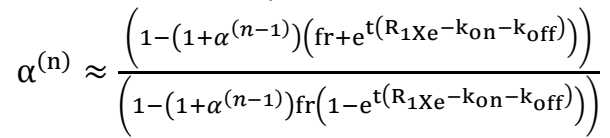

With sufficient delay time for free chemical exchange, we obtained $\mathrm{e}^{\mathrm{t}\left(\mathrm{R}_{1} \mathrm{Xe}-\mathrm{k}_{\mathrm{on}}-\mathrm{k}_{\text {off }}\right)} \approx 0$. Thus, we obtained $\alpha^{(\mathrm{n})} \approx 1$ for arbitrarily $180^{\circ}$ inversion pulse-delay number $\mathrm{n}$. We further $\operatorname{simplified} \mathrm{M}_{\mathrm{Xe}}^{(\mathrm{n})}(t)$ and $\mathrm{M}_{\mathrm{CXe}}^{(\mathrm{n})}(t)$ into the following:

$$
\begin{aligned}
& \mathrm{M}_{X e}^{(n)}(\mathrm{t}) \approx \mathrm{M}_{X \mathrm{e}}^{(\mathrm{n}-1)}(\mathrm{t}) \mathrm{e}^{-\mathrm{R}_{1 X e} \mathrm{t}_{\mathrm{p}}} \mathrm{e}^{\left(-\mathrm{R}_{1 X e}\right)}(1-2 \mathrm{fr}) \\
& \mathrm{M}_{\mathrm{CXe}}^{(\mathrm{n})}(\mathrm{t}) \approx \mathrm{M}_{\mathrm{Xe}}^{(\mathrm{n}-1)}(\mathrm{t}) \mathrm{e}^{-\mathrm{R}_{1 X e} \mathrm{t}_{\mathrm{p}} \mathrm{e}^{\left(\mathrm{t}\left(-\mathrm{R}_{1 X e}\right)\right)} \mathrm{fr}(1-2 \mathrm{fr})}
\end{aligned}
$$

Then, we obtained the magnetization expression of free Xe after the $n$th $180^{\circ}$ inverse pulse-delay unit in an on-resonance experiment, as follows:

$$
\mathrm{M}_{\mathrm{Xe} \_ \text {on }}(\mathrm{t}) \approx \mathrm{M}_{\mathrm{Xe}}^{(0)}(0) \mathrm{e}^{-\mathrm{nR}_{1 X \mathrm{Xe}}\left(\mathrm{t}_{\mathrm{p}}+\mathrm{t}\right)}(1-2 \mathrm{fr})^{\mathrm{n}}
$$

According to the definition of CEITR, we obtained the expression of CEITR, as follows:

$$
\text { CEITR }=\frac{M_{\text {Xe_on }}(\mathrm{t})}{M_{X_{-} \_o f f}(\mathrm{t})}=(1-2 \mathrm{fr})^{\mathrm{n}}
$$

Then, we achieved the ratio between host molecule bound $\mathrm{Xe}$ and free $\mathrm{Xe}$, as follows:

$$
\mathrm{fr}=\frac{1-\mathrm{CEITR}^{\frac{1}{n}}}{2}
$$

Finally, the host molecule concentration $[\mathrm{HM}]$ was quantified with the obtained $\mathrm{fr}$, the binding constant $\mathrm{K}$, and the free $\mathrm{Xe}$ concentration $[\mathrm{Xe}]^{2}$

$$
[\mathrm{HM}]=\operatorname{fr}\left(\frac{1}{\mathrm{~K}}+[\mathrm{Xe}]\right)
$$

The fr was calculated from the CEIT experiment with formula (A25). The binding constant $\mathrm{K}$ was obtained by NMR spectroscopy method as shown in the next part. The free Xe concentration was achieved by the solubility of $\mathrm{Xe}$ in water and partial pressure of $\mathrm{Xe}$ with formula $[\mathrm{Xe}]=[\mathrm{Xe}$ Solubility]·[Xe partial pressure].

\section{Section S2. Measurement of the binding constant of $\mathrm{CrA}-(\mathrm{COOH})_{6}$}

The ratio $\mathrm{fr}$ of xenon in the host to free xenon was measured from peak integrals in the direct ${ }^{129} \mathrm{Xe}$ NMR spectrum of $2.5 \mu \mathrm{M} \mathrm{CrA}-(\mathrm{COOH})_{6}$ solution (Figure S3). Noting that $[\mathrm{HM}]=$ [unoccupied $\left.\mathrm{HM}\right]$ 
$+[\mathrm{Xe} @ \mathrm{HM}]$, in which $[\mathrm{HM}]$ stands for the host molecule concentration, [unoccupied HM] and [Xe@HM] were the concentrations of unbound and bound xenon host molecule, respectively. We rearranged the binding constant $\mathrm{K}$ as follows ${ }^{2-3}$ :

$$
\begin{aligned}
\mathrm{K}=\frac{[\mathrm{Xe} @ \mathrm{HM}]}{[\mathrm{Xe}]([\mathrm{HM}]-[\mathrm{Xe} @ \mathrm{HM}])}=\frac{\mathrm{fr}}{[\mathrm{HM}]-\mathrm{fr}[\mathrm{Xe}]} \\
=\frac{0.0036}{2.500-0.0036 \times\left(4.4 \times 1000 \times\left(\frac{65.7}{14.696} \times 0.02\right)\right)} \mu \mathrm{M}^{-1} \\
\approx 3322 \mathrm{M}^{-1}
\end{aligned}
$$

Using the solubility of xenon in water ${ }^{4}(4.4 \mathrm{mM} / \mathrm{atm} 297 \mathrm{~K})$ and the known amount of host added, we then calculated the binding constant $\mathrm{K}$. The direct spectrum was integrated to determine $\mathrm{fr}$, as shown below.

\section{Section S3. Analysis of the standard deviation of concentration determination}

As shown in equation (A26), the concentration of the xenon host molecule was proportional to $\mathrm{fr}$ with the known binding constant and free xenon concentration. While for fr, the standard deviation was obtained as follows:

$$
\sigma_{\text {fr }}^{2}=\left(\frac{\partial \mathrm{fr}}{\partial \text { CEITR }}\right)^{2} \sigma_{\text {CEITR }}^{2}=\left(\frac{\text { CEITR }^{\frac{1}{n}-1}}{2 \mathrm{n}}\right)^{2} \sigma_{\text {CEITR }}^{2}
$$

The relative deviation of $\mathrm{fr}$ was shown to be:

$$
\mathrm{RD}_{\mathrm{fr}}=\frac{\sigma_{\mathrm{fr}}}{\mathrm{fr}}=\frac{\operatorname{CEITR}\left(\frac{1}{n}-1\right)}{\mathrm{n}\left(1-\operatorname{CEITR}^{\frac{1}{n}}\right)} \sigma_{\text {CEITR }}
$$

The $\sigma_{\text {CEITR }}$ term was determined by the noise level during the measurement. The minimum value of term $\frac{\operatorname{CEITR}\left(\frac{1}{n}-1\right)}{n\left(1-\operatorname{CEITR} \frac{1}{n}\right)}$ could be obtained when CEITR $=\left(1-\frac{1}{n}\right)^{n} \rightarrow e^{-1} \approx 0.368$. Thus, when CEITR is $\mathrm{e}^{-1}$ or close to it, the minimum $\mathrm{RD}_{\mathrm{fr}}$ will be obtained. To obtain a minimum $\mathrm{R}_{\mathrm{fr}}$, an optimal cycle number $\mathrm{N}_{\text {opt }}$ can be calculated using a measured CEITR under cycles number $\mathrm{N}$ by the formula below:

$$
\mathrm{N}_{\mathrm{opt}}=-\frac{\mathrm{N}}{\ln (\mathrm{CEITR})}
$$




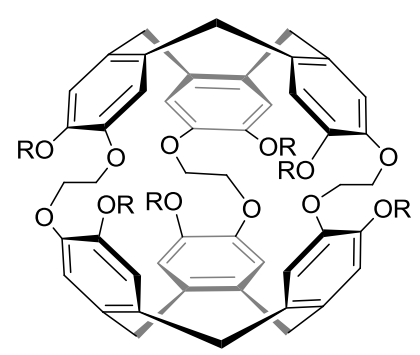

Figure S1. The structure of $\mathrm{CrA}-(\mathrm{COOH})_{6} . \mathrm{R}=-\mathrm{CH}_{2} \mathrm{COOH}$. 


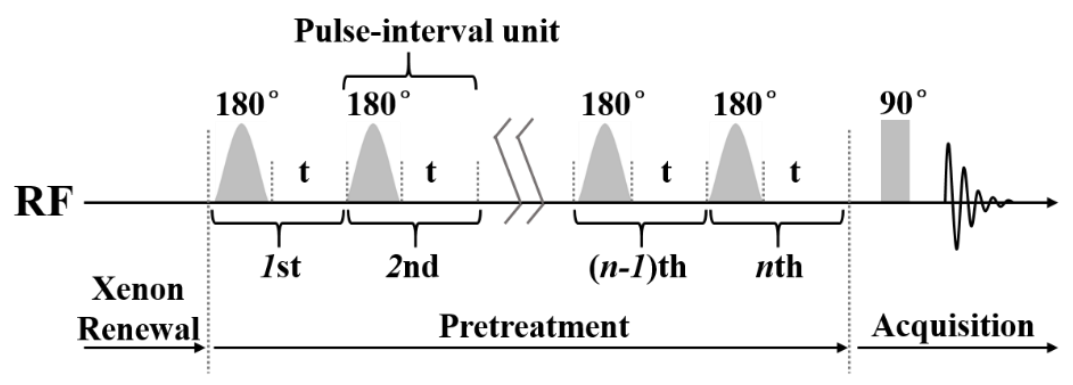

Figure S2. The CEIT pulse sequence diagram. The $180^{\circ}$ pulses are selective inversion pulses, $t$ is the interval time between $180^{\circ}$ pulses, and $\mathrm{n}$ is the number of pulse-interval unit repeat time. 


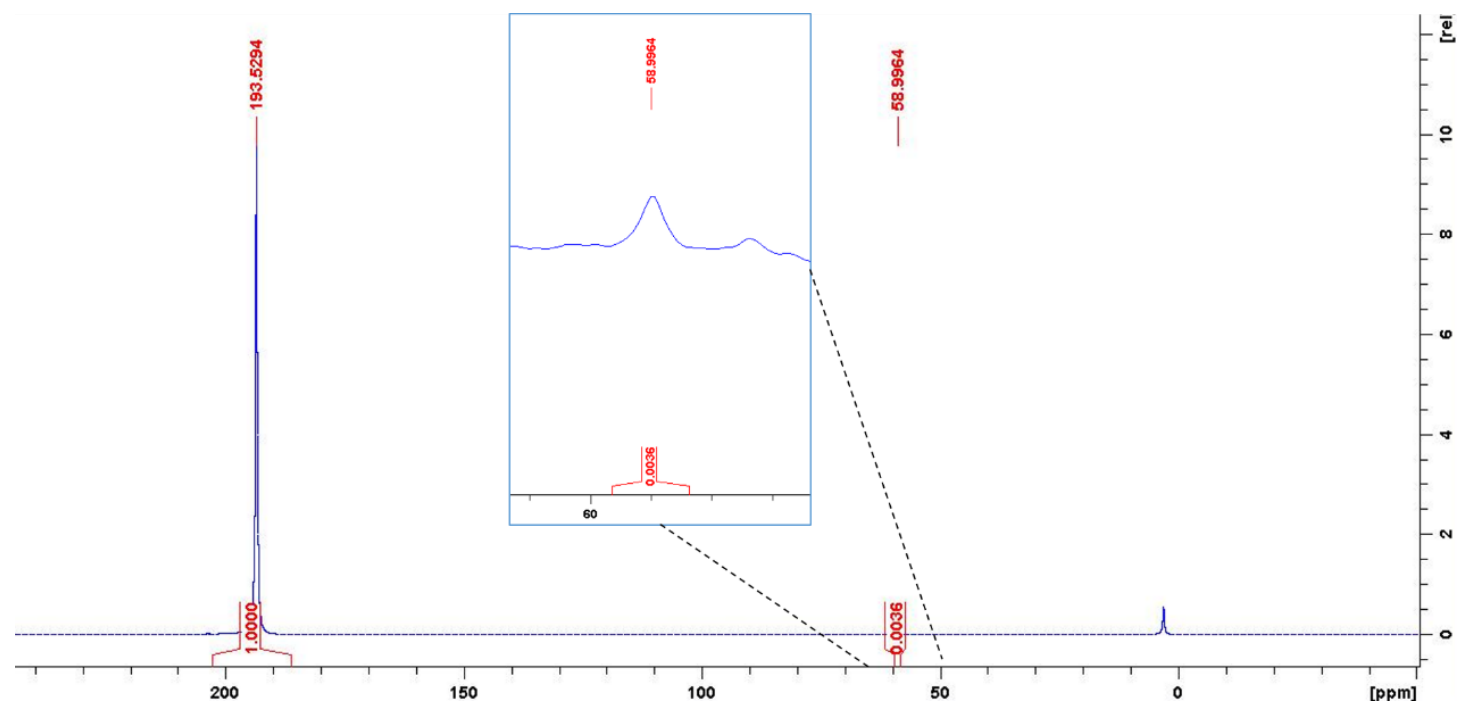

Figure S3. $2.5 \mu \mathrm{M} \mathrm{CrA}-(\mathrm{COOH}) 6{ }^{129} \mathrm{Xe} N \mathrm{NR}$ spectroscopy with NS=64. 


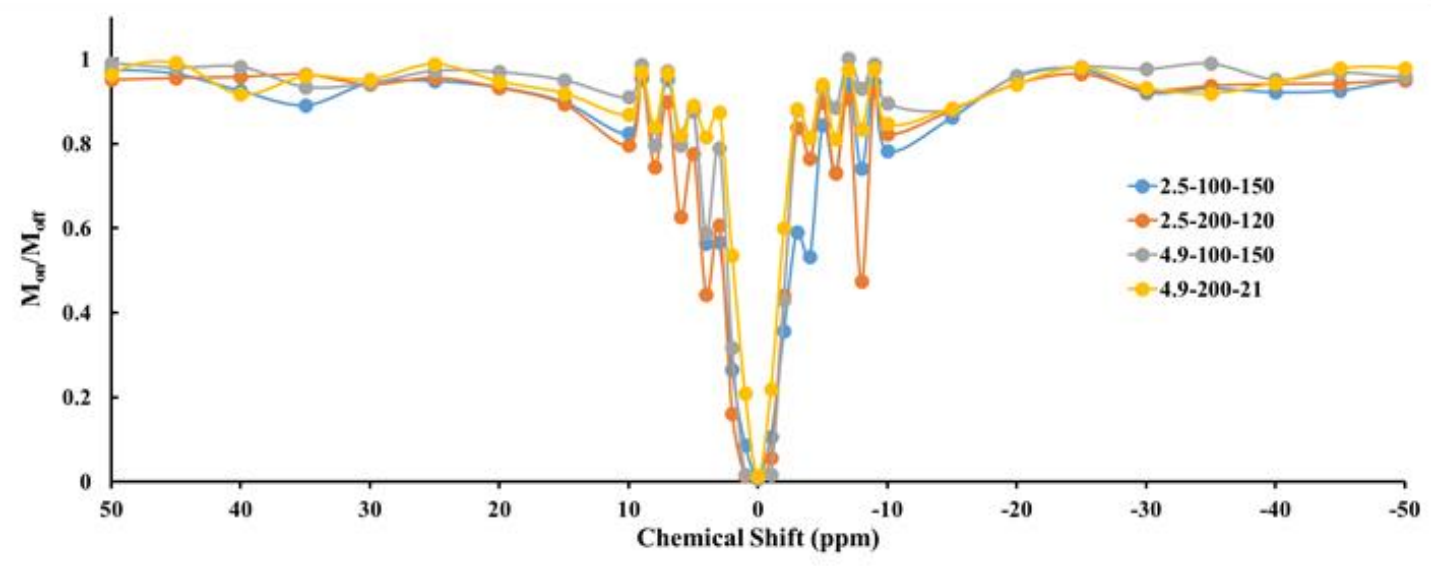

Figure S4. The local CEIT z-spectra achieved with different experimental settings (blue: $2.5 \mu \mathrm{M}$, 100 cycles, $t_{d}=150 \mathrm{~ms}$; red: $2.5 \mu \mathrm{M}, 200$ cycles, $t_{d}=120 \mathrm{~ms} ;$ grey: $4.9 \mu \mathrm{M}, 100$ cycles, $t_{d}=150 \mathrm{~ms}$; orange: $2.5 \mu \mathrm{M}, 200$ cycles, $t_{d}=21 \mathrm{~ms}$ ). 


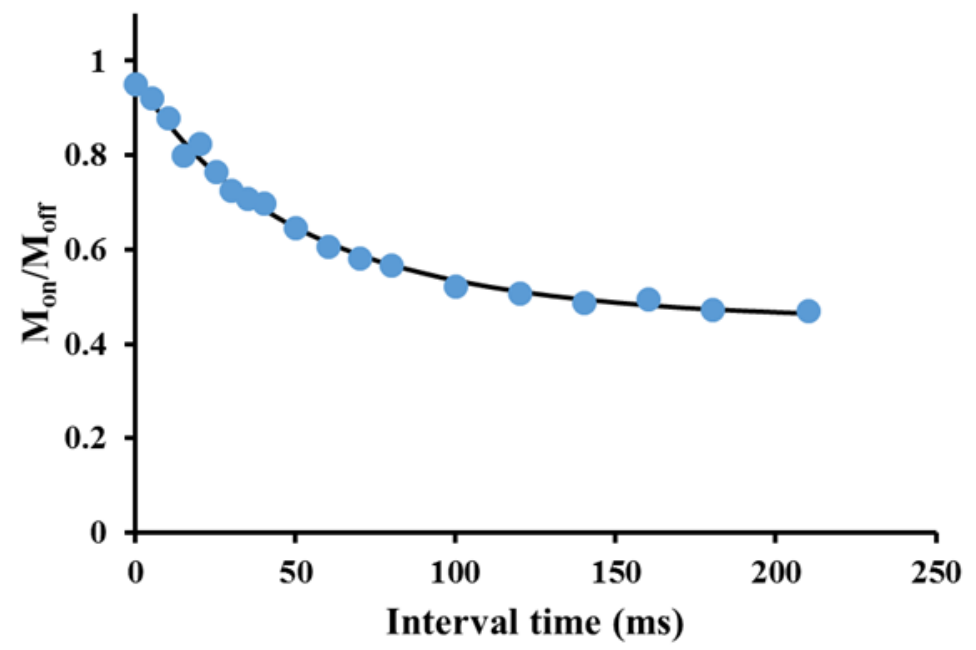

Figure S5. The exchange rate fitting of $2.5 \mu \mathrm{M} \mathrm{CrA}-(\mathrm{COOH})_{6}$ at $297 \mathrm{~K}$ and 65.7 psi. The fitted exchange rate $k$ is about $15.35 \mathrm{~Hz}$ using the following equation $\left(\mathrm{y}=\mathrm{A}\left(1-2 B\left(1-e^{-k(t-C)}\right)\right)^{100}\right.$, data: blue squares, fit: black line). 


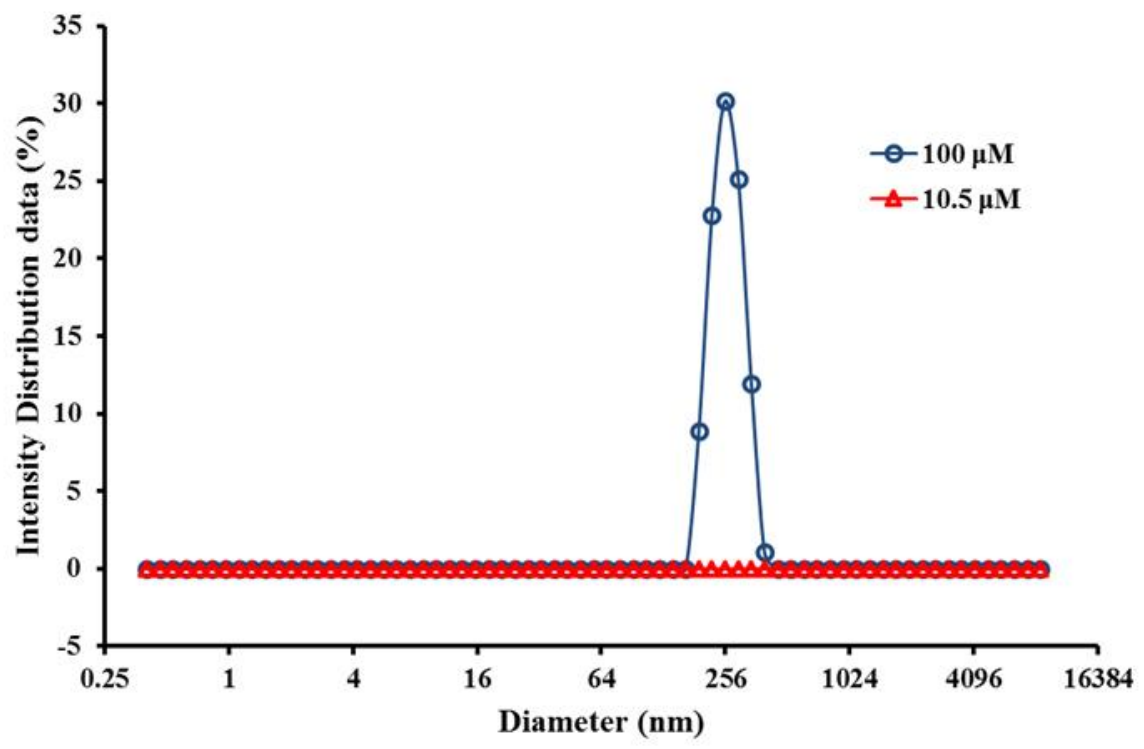

Figure S6. DLS data showing size distribution by number in aqueous solution at $298 \mathrm{~K}$ of 100.0 $\mu \mathrm{M} \mathrm{CrA}-(\mathrm{COOH})_{6}($ average diameter $=357.0 \mathrm{~nm}, \mathrm{Pdl}=0.463)$ and $10.5 \mu \mathrm{M} \mathrm{CrA}-(\mathrm{COOH})_{6}$ (no aggregation observed). 


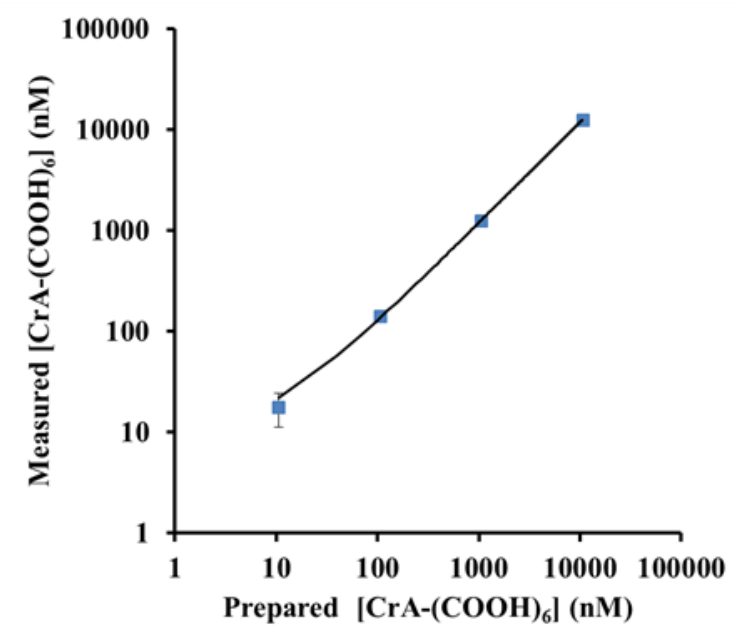

Figure S7. The comparison of the measured $\mathrm{CrA}-(\mathrm{COOH})_{6}$ concentrations and prepared CrA$(\mathrm{COOH})_{6}$ concentrations ranges from $10.5 \mathrm{nM}$ to $10.5 \mu \mathrm{M}$. The number of cycles $N$ of concentration from low to high are $800,800,200$ and 50 , respectively. The measured $\operatorname{CrA}-(\mathrm{COOH})_{6}$ concentrations agree well with the prepared $\operatorname{CrA}-(\mathrm{COOH})_{6}$ concentrations $(y=1.182 x+9.654$, $\left.R^{2}=1.000\right)$. 


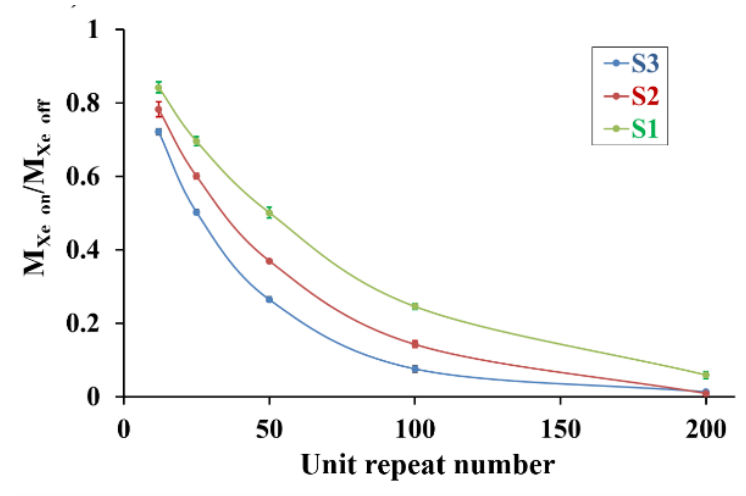

Figure S8. The CEIT effect of sample S1 (green), S2 (red), S3 (blue) under variable unit repeat numbers $\left(200,100,50,25\right.$, and 12 cycles, $\left.t_{d}=150 \mathrm{~ms}\right)$.

\section{SI References}

(1) Korchak, S.; Kilian, W.; Schröder, L.; Mitschang, L. Design and Comparison of Exchange Spectroscopy Approaches to Cryptophane-Xenon Host-Guest Kinetics. J. Magn. Reson. 2016, 265, 139-145.

(2) Kunth, M.; Witte, C.; Schröder, L. Quantitative Chemical Exchange Saturation Transfer with Hyperpolarized Nuclei (qHyper-CEST): Sensing Xenon-Host Exchange Dynamics and Binding Affinities by NMR. J. Chem. Phys. 2014, 141 (19), 194202.

(3) Tyagi, R.; Witte, C.; Haag, R.; Schröder, L. Dendronized Cryptophanes as WaterSoluble Xenon Hosts for Xe-129 Magnetic Resonance Imaging. Org. Lett. 2014, 16 (17), 4436-4439.

(4) Clever, H. L.; Battino, R. Krypton, Xenon and Radon: Gas Solubilities 1979. 